\title{
Diagnostic value of strain elastography for differentiating benign and malignant soft tissue masses
}

\author{
SHILIN LI ${ }^{1}$, LINGFANG LIU ${ }^{1}$ and GUORONG LV ${ }^{2}$ \\ ${ }^{1}$ Department of Ultrasound, Second Affiliated Hospital of Fujian Medical University; ${ }^{2}$ Department of Clinical Medicine, \\ Quanzhou Medical College, Quanzhou, Fujian 362000, P.R. China
}

Received August 11, 2015; Accepted March 30, 2017

DOI: $10.3892 / 01.2017 .6385$

\begin{abstract}
The aim of the current study was to investigate the importance of strain elastography (SE) in the differential diagnosis of benign and malignant soft tissue masses. SE was adopted to examine 61 patients with superficial masses, classify their elastic scores and assess their strain ratios (SRs) between the masses and the surrounding structures. Significantly increased SR values and elastic scores were observed in the malignant masses compared with the benign masses $(5.42 \pm 3.47$ vs. $1.80 \pm 2.10, \mathrm{P}<0.001 ; 3.13 \pm 0.34$ vs. $2.03 \pm 0.99$, $\mathrm{P}<0.001)$. Area under receiver operating characteristic curve values of the SRs and elastic scores were $0.87(\mathrm{P}<0.001)$ and $0.805(\mathrm{P}=0.001)$, respectively. With an $\mathrm{SR}$ of $>2.295$ as the optimal threshold value, the sensitivity, specificity and positive and negative predictive values for diagnosing a malignant mass were 93.8, 80.5, 65.2 and $97.1 \%$, respectively; whilst using an elastic score of $\geq 3$ as the optimal threshold value, the sensitivity, specificity and positive and negative predictive values for diagnosing a malignant mass were 100, 51.6, 51.6 and $100 \%$, respectively. SR values and elastic scores were significantly different between the malignant and benign soft tissue masses. Therefore, SE may be used to effectively differentiate between malignant and benign soft tissue masses.
\end{abstract}

\section{Introduction}

Superficial soft tissue masses frequently occur and primarily manifest as benign lesions (including lipoma and hemangioma) that typically do not require treatment. Although malignant masses are rare, prompt surgical resections are required following the confirmation of a diagnosis (1). Therefore, differentiating between benign and malignant masses is important to prevent delays in the treatment of the malignant

Correspondence to: Dr Guorong Lv, Department of Clinical Medicine, Quanzhou Medical College, 2 Anji Road, Quanzhou, Fujian 362000, P.R. China

E-mail: 1slqz@139.com

Key words: soft tissue mass, elastography, strain elastography, pathology, diagnosis masses and avoid unnecessary surgical treatments for the benign masses (2). As the most effective method, pathological diagnosis is typically obtained from a needle biopsy. However, it is an invasive inspection that is uncomfortable for patients and impractical for all types of soft tissue masses (3). Ultrasound is the primary examination method for superficial soft tissue masses to confirm their size, location and association between the masses and the surrounding structures. Through observations of the borders of the tissue masses, internal echo characteristics and internal blood flow signals, ultrasounds may provide a preliminary diagnosis that is inaccurate (4). Stiffness of the tissue structures may be accessed using ultrasound strain elastography (USE) (5), which is an effective tool for differentiating malignant and benign masses (6). Stiffness of a malignant tumor is typically higher compared with a benign tumor. Previously, the differential diagnosis was primarily based on palpations by the physicians, which was indirect and could be limited in patients with obesity, mass sizes and depths, and physicians' experiences (7). Following the first application at the end of the last century, USE has been widely accepted as an effective method for differentiating between malignant and benign tumors, in particular the differential diagnosis for breast cancer (8). In addition, USE has been successfully applied in the diagnoses of thyroid, liver and kidney tumors (9-11). However, differentiation of malignant and benign soft tissue masses using USE has rarely been investigated (12). The current study aimed to assess the importance of strain elastography (SE) for the differentiation of malignant and benign soft tissue masses.

\section{Materials and methods}

Patients and treatments. The present study was approved by the Ethics Committee of the Second Affiliated Hospital of Fujian Medical University and written informed consent was obtained from all patients. Between October 2012 and November 2014, 66 patients (34 males and 32 females) admitted at the Second Affiliated Hospital of Fujian Medical University (Quanzhou, China) due to palpable superficial masses were enrolled onto the current study. The mean age was $45.9 \pm 15.9$ years (range, 6-74). Conventional ultrasound and USE were performed on all patients. Surgical resections were sequentially performed on 48 patients with normal clinical histopathology carried out by professionals 

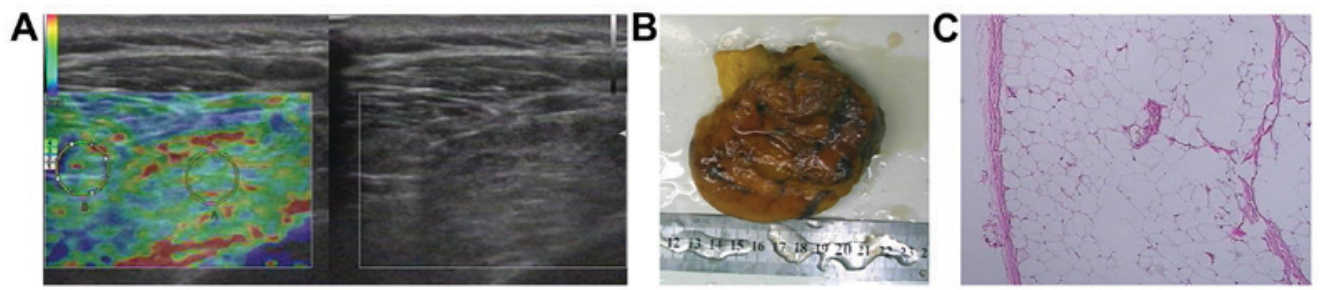

Figure 1. Left shoulder intermuscular lipomyoma of a 27-year-old woman. (A) Sonoelastography identified red and green color with elasticity score of 1 and strain ratio of 0.77 , which was confirmed by (B) excision and (C) pathology (hematoxylin and eosin staining).
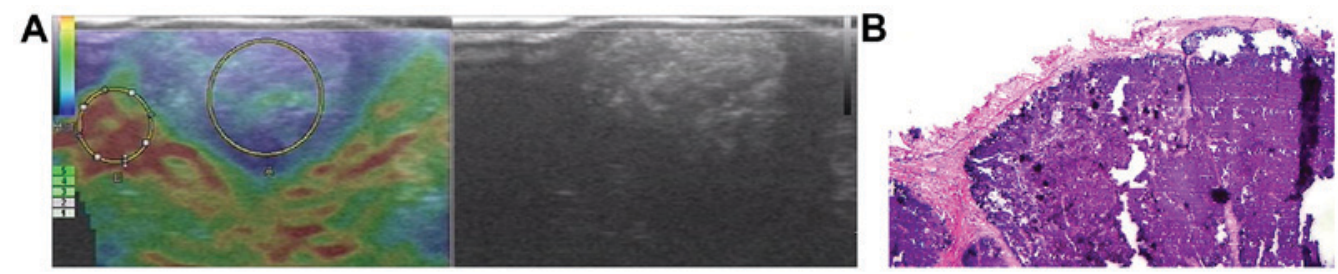

Figure 2. Subcutaneous calcinosis in the left hip of a 21-year-old man. (A) Sonoelastography identified dominantly blue color with elasticity score of 3 and strain ratio of 9.40, which was confirmed by (B) pathology (hematoxylin and eosin staining).
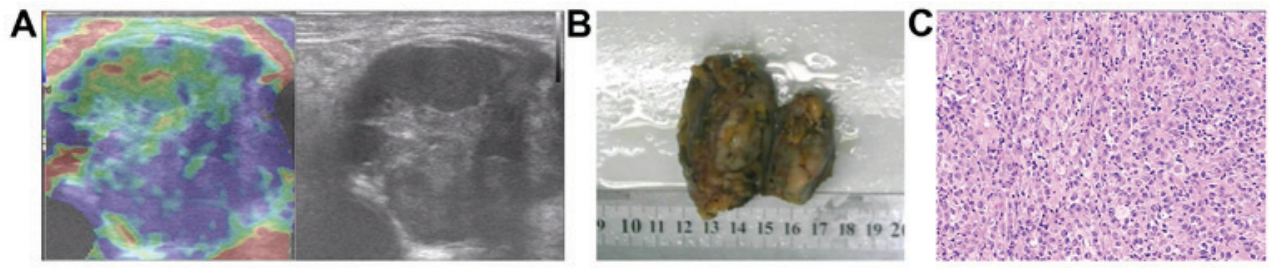

Figure 3. Malignant melanoma in the right inguen of a 67-year-old man. (A) Sonoelastography identified primarily blue and less green color with elasticity score of 3 and strain ratio of 12.70, which was confirmed by (B) excision and (C) pathology (hematoxylin and eosin staining).

of our hospital $(0.2 \%$ hematoxylin for 5 min and $0.5 \%$ eosin stained for $2 \mathrm{~min}$ ), whilst no treatments were administered to 13 patients with benign masses following a comprehensive diagnosis. During the $>1$-year follow-up period, no significant alterations in the masses were observed. In total 5 patients were lost to follow-up and therefore excluded from the study. The ultrasound instrument was VISION Preirus (Hitachi, Ltd., Tokyo, Japan) with a 3-13 MHz linear transducer and the examination was performed by a radiologist with 10 years of experience in ultrasound examination and $>5$ years of expertise in USE examination. Based on the locations of the masses, different patient positions were adopted to ensure that the body surfaces, where the masses were located, were parallel to the examination table. Gray-scale and color Doppler examinations were initially performed to observe the locations and sizes of the masses and their association with the adjacent structures and subsequently, the SE mode was initiated. The probe was repeatedly and mildly pressed then released to obtain the elastic images. Every site was examined 3 times and the images were captured for further analysis.

USE analysis. Colors of the images represented different strain rates in a decreasing order from red to green to blue. Higher strain rates indicated greater deformation tendencies and lower stiffness of the tissues. Based on the image colors, tissue elasticity was classified into 4 scores representing different stiffness: score 1, completely red or green; score 2 , blue and green, with green as the dominant color; score 3 , blue and green, with blue as the dominant color; and score 4 , completely blue. Strain rates of samples from inside and outside the masses were measured to calculate the strain ratios (SRs).

Statistical analysis. SPSS software (version 19.0; IBM SPSS, Armonk, NY USA) was adopted for the statistical analysis. The non-parametric test was used to compare the elastic scores and SR values between the benign and malignant masses. The receiver operating characteristic (ROC) curve of elastic score and $\mathrm{SR}$ value was generated to calculate the area under the curve (AUC), determine the optimal threshold values and measure the sensitivity and specificity. $\mathrm{P}<0.05$ was considered to indicate a statistically significant difference.

\section{Results}

Of the 61 patients with complete follow-up data, 31 had benign soft tissue masses, including 11 lipomas (Fig. 1), 6hemangiomas, 4 fibromas, 4 inflammatory masses (Fig. 2), 3 epidermoid cysts and 3 neurofibromas, and 13 other benign masses confirmed by the unchanged status during the $>1$-year follow-up. In total 17 patients had malignant masses, comprising of 10 metastatic carcinomas, 3 lymphomas, 2 malignant melanomas (Fig. 3), a liposarcoma and a myeloma. The elastic scores and SR 


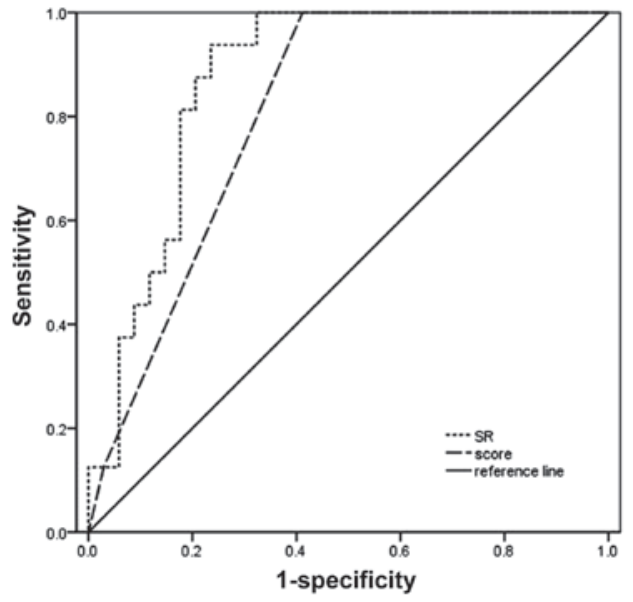

Figure 4. Receiver-operating characteristic curves for SR and scores of soft tissue mass elastography. SR, strain ratio.

values of the benign and malignant masses were $2.03 \pm 0.99$ and $3.13 \pm 0.34(\mathrm{P}<0.001)$, respectively, and $1.80 \pm 2.10$ and $5.42 \pm 3.47(\mathrm{P}<0.001)$, respectively. Area under receiver operating characteristic curve (AUROC) values of the SRs and elastic scores were $0.87(\mathrm{P}<0.001 ; 95 \%$ confidence interval of $0.775-0.968)$ and $0.805(\mathrm{P}=0.001 ; 95 \%$ confidence interval of 0.688-0.922), respectively (Fig. 4). There were no significant differences identified between the AUROCs of the 2 methods ( $P>0.05)$. Using analysis of the ROC data, the optimal SR threshold value for determining a malignant mass was 2.295, with a sensitivity of $93.8 \%$, specificity of $80.5 \%$, positive predictive value of $65.2 \%$ and negative predictive value of $97.1 \%$, whereas adopting an elastic score $\geq 3$ (Fig. 5A and B) as the optimal threshold value, the sensitivity, specificity, positive predictive value and negative predictive value for diagnosing a malignant mass were 100,51.6, 51.6 and 100\%, respectively.

\section{Discussion}

By applying pressure to the inspection sites, USE acquires response information resulting from the pressure and determines the tissue stiffness. As malignant tumors are typically harder compared with benign tumors, USE may be used to differentiate between them (13). The two most frequently used USE methods are SE and shear wave elastography (SWE) (14). SE acquires the deformation information of the tissues under pressure, with greater deformations indicating lower tissue stiffness and less deformations representing greater tissue stiffness, and presents the results in different colors or differing degrees of brightness. SWE obtains the shear wave information from the tissues under pressure, with faster propagation velocities of shear wave indicating greater tissue stiffness, and also presents the results in different colors or differing degrees of brightness. In addition, SWE also measures and quantifies the shear wave propagation velocities at the regions of interest, and therefore provides more information compared with SE. However, SWE is a novel technique with an inadequate number of published studies, and its advantages have not been conclusively demonstrated. Chang et al (15) and Youk et al (16) compared the importance of SWE and SE for differentiating between malignant and benign breast masses, and did not identified any significant differences between the AUROCs of these 2 methods. Carlsen et al (17) assessed the elastic scores of targets with different diameters and depths using SE, SWE and strain histogram, and observed that SE and strain histogram AUCs were higher compared with the SWE AUC, and target diameter influenced all 3 methods, whilst depth only influenced shear-wave velocity. Mass depths do not significantly differ in small organs, including the thyroid (18), but in the current study, masses had greater depth ranging from the subcutaneous layer to the muscular layer, which may result in an increased frequency in errors in the SWE examination. As SE is primarily unaffected by the mass depths, it is potentially advantageous compared with SWE in the differentiation of soft tissue masses.

Riishede et al (12) applied SE to predict malignancy in 60 patients with a total of 61 soft tissue tumors and identified significant differences between the mean SR values for malignant and benign tumors, with significantly higher SR in the malignant tumors, but no significant differences were observed for strain histograms or elastic scores. The results of the present study indicated significant differences in the SR values and elastic scores between the malignant and benign masses. Setting an SR of $>2.295$ and an elastic score of $\geq 3$ as thresholds was highly sensitive for the diagnosis of a malignant mass (sensitivities, 93.8 and 100\%, respectively). If a mass is diagnosed as benign by the 2 methods, possibility of malignancy may be excluded with the aid of two-dimensional and color Doppler examinations, and needle biopsies may be avoided. The specificities of SR and elastic score for diagnosing a malignant tumor were comparatively low (specificities were 80.5 and $51.6 \%$, respectively), which may be as certain benign masses also have high stiffness. For instance, a particular patient with calcinosis has an SR value of 9.4 and elastic score of 3, whilst another patient with epidermoid cyst
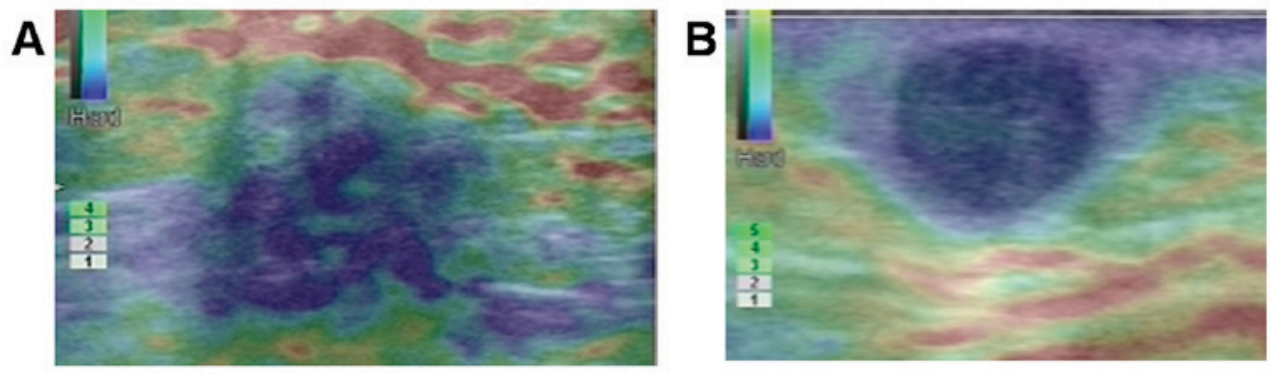

Figure 5. Strain elastographic scores of soft tissue masses. (A) Score 3, blue and green, with blue as the dominant color; (B) score 4, completely blue. 
complicated by foreign body giant cell reaction had SR value of 4.4 and elastic score of 3 . The resected tissue samples from these patients exhibited high stiffness.

The current study has certain limitations. As the origins of the soft tissue tumors are diverse, only the elasticity between the malignant and benign masses were compared and not the masses from different pathological types due to the small sample size. Further studies with larger sample sizes are required. The degree of motion is also an effective way to differentiate between the malignancy and benignancy of a mass. During the physical examination, the degree of motion may be determined by palpations, which is not sufficiently achieved by USE. This disadvantage of USE highlights the simplicity and effectiveness of palpation in clinical practice.

In conclusion, SR values and elastic scores of the malignant soft tissue masses were significantly higher compared with those of the benign tissues. With a high sensitivity, SE may be used to differentiate between the malignant and benign soft tissue masses. Setting an SR value of $>2.295$ and elastic score of $\geq 3$ as the threshold for diagnosing a malignant tumor, is highly sensitive but not sufficiently specific.

\section{References}

1. Kransdorf MJ: Benign soft-tissue tumors in a large referral population: Distribution of specific diagnoses by age, sex, and location. AJR Am J Roentgenol 164: 395-402, 1995.

2. Kumral TL, Yildirim G, Önol SD, Ataç E, Uyar Y and Coşkun ZÜ: Real-time ultrasound elastography for the differentiation of malignant and benign masses in the head and neck. J Craniofac Surg 25: 1971-1974, 2014.

3. Manaster BJ: Soft-tissue masses: Optimal imaging protocol and reporting. AJR Am J Roentgenol 201: 505-514, 2013.

4. Kwok HC, Pinto CH and Doyle AJ: The pitfalls of ultrasonography in the evaluation of soft tissue masses. J Med Imaging Radiat Oncol 56: 519-524, 2012.

5. Hahn S, Lee YH, Lee SH and Suh JS: Value of the strain ratio on ultrasonic elastography for differentiation of benign and malignant soft tissue tumors. J Ultrasound Med 36: 121-127, 2017.

6. Sun J, Cai J and Wang X: Real-time ultrasound elastography for differentiation of benign and malignant thyroid nodules: A meta-analysis. J Ultrasound Med 33: 495-502, 2014.

7. Dargar S, Akyildiz AC and De S: Development of a soft tissue elastography robotic arm (STiERA).Stud Health Technol Inform 220: 77-83, 2016.
8. Kim YS, Park JG, Kim BS, Lee CH and Ryu DW: Diagnostic value of elastography using acoustic radiation force impulse imaging and strain ratio for breast tumors. J Breast Cancer 17: 76-82, 2014.

9. Choi WJ, Park JS, Koo HR, Kim SY, Chung MS and Tae K: Ultrasound elastography using carotid artery pulsation in the differential diagnosis of sonographically indeterminate thyroid nodules. AJR Am J Roentgenol 204: 396-401, 2015.

10. Lu Q, Ling W, Lu C, Li J, Ma L, Quan J, He D, Liu J, Yang J, Wen T, et al: Hepatocellular carcinoma: Stiffness value and ratio to discriminate malignant from benign focal liver lesions. Radiology 275: 880-888, 2015.

11. Göya C, Daggulli M, Hamidi C, Yavuz A, Hattapoglu S, Cetincakmak MG and Teke M: The role of quantitative measurement by acoustic radiation force impulse imaging in differentiating benign renal lesions from malignant renal tumours. Radiol Med 120: 296-303, 2015.

12. Riishede I, Ewertsen C, Carlsen J, Petersen MM, Jensen F and Nielsen MB: Strain elastography for prediction of malignancy in soft tissue tumours-preliminary results. Ultraschall Med 36: 369-374, 2015.

13. Onur MR, Poyraz AK, Bozgeyik Z, Onur AR and Orhan I: Utility of semiquantitative strain elastography for differentiation between benign and malignant solid renal masses. J Ultrasound Med 34: 639-647, 2015.

14. Kwak JY and Kim EK: Ultrasound elastography for thyroid nodules: Recent advances. Ultrasonography 33: 75-82, 2014.

15. Chang JM, Won JK, Lee KB, Park IA, Yi A and Moon WK: Comparison of shear-wave and strain ultrasound elastography in the differentiation of benign and malignant breast lesions. AJR Am J Roentgenol 201: W347-W356, 2013.

16. Youk JH, Son EJ, Gweon HM, Kim H, Park YJ and Kim JA: Comparison of strain and shear wave elastography for the differentiation of benign from malignant breast lesions, combined with B-mode ultrasonography: Qualitative and quantitative assessments. Ultrasound Med Biol 40: 2336-2344, 2014.

17. Carlsen JF, Pedersen MR, Ewertsen C, Săftoiu A, Lönn L, Rafaelsen SR and Nielsen MB: A comparative study of strain and shear-wave elastography in an elasticity phantom. AJR Am J Roentgenol 204: W236-W242, 2015.

18. Yamanaka N, Kaminuma C, Taketomi-Takahashi A and Tsushima Y: Reliable measurement by virtual touch tissue quantification with acoustic radiation force impulse imaging: Phantom study. J Ultrasound Med 31: 1239-1244, 2002. 